\title{
Capsule Commentary on Berkowitz et al., Medically Tailored Meal Delivery for Diabetes Patients with Food Insecurity: a Randomized Cross-Over Trial
}

\author{
Deidra C. Crews, MD, SCM ${ }^{1,2,3}$ \\ 'Division of Nephrology, Department of Medicine, Johns Hopkins University School of Medicine, Baltimore, MD, USA; ${ }^{2}$ Johns Hopkins Center for \\ Health Equity, Johns Hopkins Medical Institutions, Baltimore, MD, USA; ${ }^{3}$ Welch Center for Prevention, Epidemiology and Clinical Research, Johns \\ Hopkins Medical Institutions, Baltimore, MD, USA.
}

J Gen Intern Med 34(3):446

DOI: $10.1007 / \mathrm{s} 11606-018-4812-0$

(c) Society of General Internal Medicine 2019

$\mathrm{T}$ his clinical trial by Berkowitz et al. ${ }^{1}$ tested whether a medically tailored meal delivery program could improve diet quality among food-insecure individuals with uncontrolled type 2 diabetes. Using a cross-over design, they assigned 44 participants to either immediate receipt of meal delivery for 12 weeks, followed by 12 weeks of follow-up while not receiving the intervention, or delayed receipt of the intervention for 12 weeks, followed by 12 weeks of meal delivery. During the intervention, participants received onceweekly delivery of 10 refrigerated and/or frozen meals that were prepared under the guidance of a dietitian to be suitable for persons with diabetes and also took into consideration 17 other medical conditions that an individual participant might have that would warrant specific dietary modifications. The authors found that the intervention led to improved dietary quality and food insecurity, and reduced hypoglycemia. While not a primary outcome of the study, the mental health component of health-related quality of life also improved during the intervention phase.

Limitations of the study include its modest sample size and relatively short follow-up. Additionally, the study outcome assessors were not masked to participants' treatment order, and although food was only provided for the participants, and not others in the household, participants' consumption of the delivered foods during the intervention was not assessed. The latter would be important for understanding the "delivered dose" of the healthy meals that led to favorable outcomes, which might inform future interventions. Patient views of medically tailored meals were also not reported and would be important to understand as some patients may prefer interventions facilitating their independent purchase of healthy foods $^{2}$ as opposed to being provided specific meals.

For clinicians, researchers, and public health professionals, this study provides evidence that medically tailored meals might be an effective approach for addressing food insecurity among patients with diabetes. Long-term studies are needed to more fully elucidate the clinical impact(s) and related costs of medically tailored meals in this and other populations with diet-sensitive chronic conditions, and how its effects may contrast with or compliment income transfer ${ }^{3}$ and dietary education programs.

Corresponding Author: Deidra C. Crews, MD, ScM; Division of Nephrology, Department of Medicine, Johns Hopkins University School of Medicine, Baltimore, MD, USA (e-mail: dcrews1@jhmi.edu).

\section{Compliance with Ethical Standards:}

Conflict of Interest: The author declares that she has no conflict of interest.

Publisher's Note: Springer Nature remains neutral with regard to jurisdictional claims in published maps and institutional affiliations.

\section{REFERENCES}

1. Berkowitz SA, Delahanty LM, Terranova J, Steiner B, Ruazol MP, Singh R, Shahid NN, Wexler DJ. Medically tailored meal delivery for diabetes patients with food insecurity: a randomized cross-over trial. J Gen Intern Med. https://doi.org/10.1007/s11606-018-4716-Z

2. Leung CW, Musicus AA, Willet tWC, Rimm EB. Improving the nutritional impact of the Supplemental Nutrition Assistance Program: perspectives from the participants. Am J Prev Med. 2017;52(2S2):S193-S198.

3. Zhang FF, Liu J, Rehm CD, Wilde P, Mande JR,Mozaffarian D. Trends and disparities in diet quality among US adults by Supplemental Nutrition Assistance Program participation status. JAMA Netw Open. 2018;1(2):e180237.

Published online January 8, 2019 